\title{
Wnt Signaling in the Tumor Microenvironment
}

\author{
Yongsheng Ruan ${ }^{1,2}$, Heather Ogana1 ${ }^{1}$ EunJi Gang ${ }^{1}$, Hye Na Kim${ }^{1}$, Yong-Mi Kim ${ }^{1, *}$ \\ ${ }^{1}$ Department of Pediatrics, Division of Hematology, Oncology, Blood and Marrow Transplantation, \\ Children's Hospital Los Angeles, University of Southern California, Los Angeles, CA 90027, USA \\ ${ }^{2}$ Department of Pediatrics, Nanfang Hospital, Southern Medical University, Guangzhou, 510515, \\ China
}

\begin{abstract}
Dysregulated Wnt signaling plays a central role in initiation, progression and metastasis in many types of human cancers. Cancer development and resistance to conventional cancer therapies are highly associated to the tumor microenvironment (TME), which is composed of numerous stable non-cancer cells including immune cells, extracellular matrix (ECM), fibroblasts, endothelial cells (ECs), and stromal cells. Recently increasing evidence suggests that the relationship between Wnt signaling and the TME promotes the proliferation and maintenance of tumor cells including leukemia. Here, we review the Wnt pathway, the role of Wnt signaling in different components of the TME, and therapeutic strategies for targeting Wnt signaling.
\end{abstract}

\section{Keywords}

Wnt; $\beta$-catenin; cancer; tumor microenvironment; cancer stem cell; immune cell; immune tolerance; immune evasion; extracellular matrix; fibroblast; endothelial cell; stromal cell; therapy

\section{Introduction}

The Wnt signaling pathway is a critical regulator of development in embryogenesis, tissue homeostasis, stemness control, wound repair, and malignancy[1]. Recently, it has been found that aberrant Wnt signaling is involved in the pathogenesis of cancer such as immune evasion and immunomodulation[2,3]. The microenvironment of tumor cells is composed of tumor cells, immune cells, and stromal cells, which contribute to drug resistance and survival of the turnor[4],Here, we review the relationship between aberrant Wnt signaling and the tumor microenvironment (TME), and summarize the potential targeting therapeutic strategies.

\section{Wnt Signaling Pathway}

The Wnt signaling pathway has been extensively studied and reviewed[1, 5, 6]. In general, there are three pathways: the canonical Wnt pathway, the non-canonical Wnt-planar cell polarity (PCP) pathway, and the non-canonical Wnt-calcium $\left(\mathrm{Ca}^{2+}\right)$ pathway.

*Correspondence: ymkim@chla.usc.edu. 


\subsection{The Canonical Pathway}

The canonical pathway relies on cytoplasmic $\beta$-catenin stabilization [7]. However, $\beta$-catenin is a highly unstable protein. In the absence of Wnt ligands, $\beta$-catenin is part of the $\beta$-catenin destruction complex composed of adenomatous polyposis coli (APC), AXIN, casein kinase $1 a(\mathrm{CK} 1 \alpha)$ and glycogen synthase kinase 3 (GSK-3), which catalyzes the phosphorylation of $\beta$-catenin at its $\mathrm{N}$ terminus and tags ubiquitin protein ligase $\beta-$ Trcponto $\beta$ catenin. After poly-ubiquitination, $\beta$-catenin is then degraded byproteasomes[8]. Upon the binding of Wnt ligand to Frizzled (FZD) receptors, which is a G-protein-coupled receptor (GPCR) with seven-transmembrane domains[9], and the co-receptor low density lipoprotein receptor-related protein 5/6(LRP5/6)[10]. Next, disheveled segment polarity protein (DVL) is recruited intracellularly as a platform for AXIN to interact with the cytoplasmic domain of LRP5/6.This interaction disassembles the destruction complex, therefore resulting in stabilization of $\beta$-catenin. Ultimately $\beta$-catenin accumulates and translocates to the nucleus, where it can bind to the transcription factor T-cell factor/lymphoid enhancer factor(TCF/LEF), and recruit the transcriptional Kat3 co-activators p300 and/or CREBbinding protein(CBP) to transcribe Wnt target genes[11]. In contrast, TCF is in an inactive state as the consequence of binding to the repressor Groucho. The genes activated by Wnt have important functions for many processes in oncogenesis and development such as self-renewal, differentiation, proliferation, and metastasis[12, 13]. (Figure 1)

\subsection{The Non-canonical Pathways}

The non-canonical Wnt pathways coexist with the canonical pathway and are $\beta$-cateninindependent[6]. In the non-canonical Wnt-planar cell polarity (PCP) pathway, the Wnt ligand binds to Frizzled receptors and activates the small GTPasesRhoA and Ras-related C3 botulinum toxin substrate 1 (RAC1) via activation of DVL. RhoA upregulates Rho kinase while activated RAC1 enhances c-Jun N-terminal kinase (JNK) expression, triggering the expression of downstream target genes[5]. The non-canonical Wnt-Ca2+ pathway is initiated by $\mathrm{G}$ protein-mediated phospholipase C (PLC) activation, which induces the influx of calcium. Calcium acts as a second messenger and further activates of downstream proteins such as calmodulin-dependent protein kinase II (CAMKII) and protein kinase C (PKC), resulting in cell migration[14]. Wnt signaling contributes to the stabilization of proteins other than $\beta$-catenin to maintain intracellular functions through these alternative pathways [15].

\section{WntSignaling and Cancer Stem Cells}

The self-renewal potential of cancer cells is described by the cancer stem cells (CSCs) in solid tumors[16]. There is adequate evidence that Wnt signaling has a vital role for the maintenance and progression of CSCs[17]. Leukemia stem cells (LSCs) share similar properties with CSC, and leukemogenesis is closely related with aberrant Wnt signaling in both leukemic cells and stromal cells in bone marrow microenvironment[18, 19]. One of the hallmarks of CSCs is to sustain long telomeres through high expression of telomerase reverse transcriptase (TERT)[20]. $\beta$-catenin directly augments TERT expression through promoter binding[21].Recent studies showed that GSK3 $\beta$, AXIN binding to tankyrase, and the SOX family transcription factors played key roles in the maintenance of CSC 
traits in breast cancers through the Wnt signaling pathway[22-24]. One target gene of Wnt is Leucine-rich repeat-containing G-protein-coupled receptor 5 (LGR5), a bona fide marker of adult stem cells in the gastrointestinal tract that acts to enhance $\mathrm{Wnt} / \beta$ catenin signaling [25]. In colorectal cancer, LGR5 has recently been found to also be a marker of colorectal CSCs[26]. Furthermore, intestinal famesoid X receptor (FXR) function is antagonized by bile acids and induces proliferation and DNA damage in LGR5-positive CSCs [27]. Colorectal cancer cell stemness was found to be enhanced by miR-372/373, a cluster of stem cell-specific microRNAs transactivated by the Wnt pathway, by repression of differentiation genes[28]. In addition, LGR5 has a vital oncogenic role in cervical cancer by upregulating Wnt signaling and promoting cervical CSC traits[29]. In non-small cell lung carcinoma, RIF1 and serine-arginine protein kinase 1 (SRPK1) promote tumor growth and CSC-like properties as positive regulators of $\mathrm{Wnt} / \beta$-catenin signaling $[30,31]$.The activation of RNA-binding motif on Y chromosome (RBMY), which is only present in male hepatocellular carcinoma, results in the augmentation of CSC traits via stimulation of Wnt-3a[32]. In summary, Wnt signaling affects several downstream targets that are important for the maintenance of CSC function.

\section{Wnt Signaling and Immune Cells}

Ample vital advancements in studying the relationship between tumors and immune system have been illuminated over the past two decades. The immune system is known to play a role not only in tumor suppression but also in cancer progression[33]. An extensive immunogenomic analysis of more than 10,000 tumors of 33 cancer types from The Cancer Genome Atlas (TCGA) found six immune subtypes_-wound healing, IFNgamma dominant, inflammatory, lymphocyte-depleted, immunologically quiet, and TGFbeta dominant - characterized by differences in TME signatures[34]. Wnt signaling not only affects the differentiation of T-cells[35-39] but also influences other immune cells such as natural killer (NK) cells[40, 41] and dendritic cells (DC)[42-44].

\subsection{Wnt Signaling in Immune Tolerance}

Some lymphocytes within the TME that infiltrate solid tumors regulate the immune tolerance of tumors. For example, $\beta$-catenin in DCs serves as a key mediator in promoting both CD4+ and CD8+ T-cell tolerance. One possible reason is that DCs can be modulated by denileukin diftitox (DD), a diphtheria toxin fragment-IL2 fusion protein, leading to upregulation of the immune tolerance-associated $\beta$-catenin pathway[45]. Inhibition of interactions between Wnt with its cognate co-receptor LRP5/6 and Frizzled suggested that LRP5 and LRP6 in DCs play a critical role in immune tolerance[46]. Another study demonstrated that $\beta$-catenin/mTOR/IL-10 signaling impairs the ability of DCs to cross-prime CD8+ T-cell immunity[43]. A recent study showed that WNT5a released from melanoma cells resulted in paracrine WNT5 $\beta$-catenin signaling in DC, leading to an increase in the immunoregulatory enzyme, indoleamine 2,3-dioxygenase-1 (IDO), which plays a vital role in tumor-mediated immune tolerization[47]. On the other hand, in colon cancer, activation of $\beta$-catenin resulted in production of $\mathrm{T}$ helper $17\left(\mathrm{~T}_{\mathrm{H}} 17\right)$ cells-mediated inflammation that promoted cancer function[48]. 


\subsection{WntSignaling in Immune Evasion}

Active canonical Wnt signaling in immune cells in the TME is a crucial cause of resistance to cancer immunotherapies called immune checkpoint inhibitors [2]. Effective recognition of tumor-associated antigens and thus eradication of cancer by cytotoxic $\mathrm{T}$ lymphocytes (CTLs) can be interfered via cytotoxic T lymphocyte-associated protein 4 (CTLA-4) or the programmed cell death 1 (PD-1) and programmed death-ligand 1 (PD-L1) pathway[49]. In melanoma, T-cell exclusion and resistance to anti-PD-L1/anti-CTLA-4 monoclonal antibody therapy were mediated via tumor-intrinsic active $\beta$-catenin signaling[50]. One of the key factors was GSK-3 which could potentially decrease CD8+ T-cell function in cancer therapy via upregulation of PD-1 expression[51]. Moreover, in a prospective genotyping of hepatocellular carcinoma clinical study, active Wnt/ $\beta$-catenin signaling was associated with lower disease control rates (DCR), shorter median progression-free survival (PFS), and shorter median overall survival (OS), even though the 31 patients were treated with immune checkpoint inhibitors[52].A new evidence was found that not only the T-cell-inflamed phenotype correlated with efficacy of immune-checkpoint blockade but also non-T-cellinflamed tumors via activation of tumor-intrinsic Wnt/ $\beta$-catenin signaling[53]. Furthermore, stroma-derived Dickkopf-1 (Dkk1) targeted $\beta$-catenin in myeloid-derived suppressor cells (MDSCs), hence exerting immune suppressive effects during tumor progression[54].

\section{Wnt Signaling and Extracellular Matrix}

The extracellular matrix (ECM) is a strikingly dynamic structure that undergoes mechanical remodeling by most tumor cells which is critical for cancer progression from a primary tumor to metastatic disease[55, 56]. Stiff ECM activates the integrin/focal adhesion kinase (FAK) pathway, which elevates the expression of members of the $\mathrm{Wnt} / \beta$-catenin pathway and in turn enhances regulation of mesenchymal stem cell differentiation and primary chondrocyte phenotype maintenance[57]. Interestingly, the expression levels of B-cell lymphoma 2 (Bcl-2) associated X protein (Bax), procaspase-3 and -9, matrix metalloproteinase 1 (MMP1), MMP3, MMP13, WNT3a, WNT5a, WNT7a and $\beta$-catenin were significantly inhibited with resveratrol (RES) in osteoarthritis chondrocytes[58]. This indicates that sirtuin 1 (Sirt1), which can be upregulated by RES, may regulate ECM degradation in RES-treated osteoarthritis chondrocytes through the Wnt/ $\beta$-catenin signaling pathway[58]. In cervical cancer, the ECM protein collagen triple helix repeat containing 1 (CTHRC1) is regulated by E6/E7, which are the early genes of the high-risk mucosal human papillomavirus type[59]. Through activation of the noncanonical Wnt/PCP signaling pathway, ultimately the E6/E7-p53-POU2F1-CTHRC1 axis promoted cervical cancer cell invasion and metastasis[59].In a urinary bladder cancer study, increased WNT7a expression was associated with metastasis and poor prognosis. Mechanically, WNT7a-mediated MMP10 activation is mediated by the canonical Wnt/ $\beta$-catenin pathway[60]. In breast cancer, deposition of type I collagen in the ECM played an important role in metastasis due to Wnt signaling causing an increase in expression of MRTF-A, which was critical for regulation of the type I collagen gene COL1A1 in breast cancer cells [61]. Moreover, MRTF-A integrated signals from the Rho-ROCK-actinandWnt/ $\beta$-catenin pathways to regulate migration-related genes including MYL9, CYR61 and IncRNA HOTAIR that in turn stimulate breast cancer cell migration[62]. In glioma, MYH10 gene silencing 
resulted in reduced expression of MTA-1, MPP-2, MMP-9 and vimentin, and increased expression of TIMP-2, E-cadherin and collagen 1 through inhibition of the Wnt/ $\beta$-catenin pathway[63]. HEmT-DCN/sLRP6, an oncolytic adenovirus (Ad) co-expressing decorin and soluble Wnt decoy receptor, eradicated excessive accumulation of ECM in pancreatic cancer via inhibition of the Wnt/ $/$-catenin signaling pathway[64]. Previously another study demonstrated a similar effect using an ECM-degrading and Wnt signal-disrupting oncolytic adenovirus (oAd/DCN/LRP)[65]. Wnt/ß-catenin activated Ewing sarcoma cells upregulated secretion of ECM proteins such as structural collagens, matricellular proteins and tenascin $\mathrm{C}$ (TNC)[66]. Hence, ECM is secreted excessively and remodeled by cancer cells via Wnt/ $\beta$-catenin pathway in the TME.

\section{Wnt Signaling and Fibroblasts}

Cancer associated fibroblasts (CAFs) are important stromal cells components in the TME that play critical roles in tumor initiation, progression and metastasis[55, 67, 68]. There have been a tremendous number of studies that identify how Wnt signaling is involved in the formation and regulation of CAFs in different tumor types. In contrast to the expression in cancer cells as a Wnt antagonist to suppress various cancers progression[69-71], the stromal expression of Dickkopf-3 (DKK3), a HSF1 effector, regulated the pro-turnorigenic behavior of CAFs via Wnt signaling and the activation of Hippo pathway transducers YAP/TAZ[72]. CAFs also promoted the stemness, metastasis, and chemoresistance of colorectal cancer cells by secreting exosomes to increase miR-92a-3p, a microRNA, activating the $\mathrm{Wnt} / \beta$-catenin pathway and inhibiting mitochondrial apoptosis by directly inhibiting FBXW7 and MOAP1[73]. Furthermore, CAFs upregulated T-lymphoma invasion and metastasis-inducing protein-1 (TIAM1), one of the Wnt-signaling associated genes, resulting in chemoresistance in colorectal cancer[74]. Moreover, desmosomal protein Plakophilin-2 encoded by $P K P 2$ gene is a target gene of $\mathrm{Wnt} / \beta$-catenin and acts to inhibit the pathway, suggesting that PKP2 plays a role in the negative feedback control of Wnt/ $\beta$-catenin in normal and colon CAFs[75]. It was found that high levels of WNT2, which are associated with a poor prognosis in colorectal cancer, binds to its putative receptor FZD8 and activates autocrine canonical Wnt signaling in CAFs, which resulted in promoting colorectal cancer progression, invasion, and metastasis[76]. In head and neck squamous cell carcinoma, CAF-derived POSTN was an upstream ligand of protein tyrosine kinase 7 (PTK7) and promoted the CSC-like phenotype via PTK7-Wnt/ $\beta$-Catenin signaling, inducing proliferation and invasion [77].Deletion of hypoxia-inducible transcription factor (HIF-1) was associated with inactivation of Wnt/ $\beta$-catenin, significantly inhibiting tumor progression and decreasing production of CAFs in the TME[78, 79]. It has been reported that overexpression of phospholipase D2 (PLD2) in colon tumors induces senescence in neighboring fibroblasts and leads to senescence-associated secretory phenotype (SASP), contributing to tumor development by Wnt pathway activation in colon cancer[80].

A 3-dimensional multi-culture tumor-CAF spheroid phenotypic screening platform was developed to profile 1,024 candidate genes for CAF-intrinsic anti-spheroid activity, in which it was found that CAF-derived products such as Wnt and the G-protein coupled-receptor OGR1 are potential therapeutic targets for colorectal cancer[81].The activation of Wnt/ $\beta$ catenin and $\mathrm{Hgf} / \mathrm{Met}$ signaling significantly mediates interactions between CSCs and CAFs 
in mammary gland tumors[82].In epithelial ovarian cancer, STAT4 overexpression induced normal omental fibroblasts to obtain CAF-like features via tumor-derived WNT7a [83].On the other hand, WNT7a-mediated fibroblast activation was not only dependent on canonical Wnt signaling but also TGF- $\beta$ receptor signaling in breast cancer[84].Interestingly, aged fibroblasts can secrete a Wnt antagonist, sFRP2, which activates a multi-step signaling cascade that results in melanoma metastasis and therapy resistance [85]. Paracrine WNT10b from p85a-deficient fibroblasts regulated breast cancer tumorigenesis and progression via TME remodeling and epithelial-to-mesenchymal transition induced by the canonical Wnt pathway[86].Also in breast cancer, the effect of oxidative stress by oxidized ataxiatelangiectasia mutated protein kinase (ATM) on aberrant CAF proliferation is regulated through ERK, PI3K-AKT, and Wnt signaling pathways[87].

\section{Wnt Signaling and Endothelial Cells}

A vascular network is imperative in tumor development and metastasis, and the endothelial cells (ECs) in the TME are highly responsive to cues to promote angiogenesis of tumorinfiltrating blood vessels. Firstly, differentiated ECs undergo phenotypic transition to mesenchymal cells through a complex process named endothelial-mesenchymal transition (EndMT)[88]. TGF- $\beta$ and Snail transcription factor are two important stimulators of EndMT via Notch and Wnt signaling pathways utilizing Frizzled-2 (FZD2), FZD9, and Wnt5B in the induction process[89]. A similar finding from oral squamous cell carcinoma reports that Wnt5B functions in EndMT and regulates the expression of Snail and Slug proteins through activation of canonical and non-canonical Wnt signaling pathways[90]. Secondly, many advances intumor-angiogenesis via Wnt signaling have been reported. Regulators of Wnt signaling including FZD7[71] and R-spondin3 play important roles in vascular endothelial cells[91].Increased non-canonical WNT5a in squamous cell lung carcinoma inhibits endothelial cell growth and motility[92], implicating itspotential asa therapeutic target of angiogenesis-related diseases[93]. In malignant glioma, increased WNT7 expression in Olig2+ oligodendrocyte precursor-like cells (OPLCs) allowed for the invasion of glioma cells in the vasculature, and inhibition of Wnt blocked invasion and enhanced the response to temozolomide therapy[94]. Microparticles (MPs) released from ovarian cancer cells mediated activation of Wnt/ $\beta$-catenin in which RAC1 and AKT were responsible for phosphorylation and nuclear translocation in ECs for neoangiogenesis[95]. Ribosomal protein s15a (RPS15A) promoted angiogenesis in primary hepatocellular carcinoma (HCC) by enhancing Wnt/ $\beta$-catenin-induced FGF18 expression, suggesting that the RPS15A/FGF18 pathway may be a target for anti-angiogenic therapy of HCC[96]. Importantly, it has been demonstrated that FZD5 - aWnt/FZD family member - was a receptor for secreted frizzled-related protein 2 (SFRP2) and mediates SFRP2induced angiogenesis through calcineurin / nuclear factor of activated T-cells cytoplasmic 3 (NFATc3) pathway in ECs[97]. Furthermore, hypoxic colorectal cancer cells secreted exosomes enriched with WNT4 and were dependent on hypoxia-inducible factor a (HIFla), and promoted the proliferation and migration of ECs through increased $\beta$-catenin nuclear translocation[98]. Interestingly, HIF- $1 \alpha$ activated via $\mathrm{Wnt} / \beta$-catenin pathway promoted vasculogenesis and angiogenesis even in normoxic conditions due to lactate released by glioma cells[99]. Other important mediators such as vascular endothelial growth factor-A 
(VEGF-A) [100], and pro-inflammatory factor TNF-a [101] were associated with activation of Wnt/ $\beta$-catenin signal pathway in ECs. On the other hand, loss of Norrin (an atypical Wnt)/FZD4 -mediated signaling in ECs created a tumor-permissive microenvironment at the earliest, preneoplastic stages of medulloblastoma[102].In addition, in colorectal cancer Norrin/FZD4 also played a vital role in the regulation of angiogenesis as well[103].

\section{Wnt and Mesenchymal Stem Cells}

The stroma of the TME is comprised of a heterogeneous population of connective tissue cells such as fibroblasts, epithelial cells, and mesenchymal stem cells (MSCs), also referred to as mesenchymal stromal cells. The bone marrow microenvironment also contains MSCs which make up the bone marrow niche. Within the niche, the MSCs function to regulate and maintain hematopoietic stem cells (HSCs) and give rise to the majority of bone marrow stromal cell lineages, including chondrocytes, osteoblasts, fibroblasts, adipocytes, endothelial cells, and myocytes[104]. Wnt signaling is important for the proliferation and differentiation of MSCs but its exact role has not yet been elucidated. Huang et al. found that in the chondrogenic differentiation of MSCs, Wnt-signaling modulator BIO activated canonical Wntsignaling and activated genes important for stemness and proliferation, while the PFK compound inhibited signaling-induced chondrogenic differentiation and inhibited $\beta$-catenin translocation into the nucleus [105]. Another study showed that by inhibiting GSK-3-the kinase which mediates phosphorylation and degradation of $\beta$-cateninupregulation of the $\mathrm{Wnt} / \beta$-catenin pathway was observed, as well as increased chondrogenic differentiation[106, 107]. These results indicate that the Wnt pathway is critical for MSC developmental function with regards to proliferation and differentiation. In terms of the role of Wnt signaling in MSCs and cancer, it has been found that in many cancers, Ror2 receptor tyrosine kinase can act as a receptor for WNT5a to mediate $\beta$-cateninindependent non-canonical Wnt signaling. The constitutive WNT5a-Ror2 signaling in MSCs promotes proliferation and aggressiveness of gastric cancer cells by enhancing expression of CXCL16, thereby activating the CXCL16-CXCR6 axis in a cell-autonomous manner [108]. One study demonstrated by qPCR that MSCs had detectable mRNA expression of all 16 Wnt ligands, with the highest expressed being WNT5a, WNT3, WNT10a, and WNT7b[109]. The authors found that co-culture of acute lymphoblastic leukemia (ALL) with MSCs provided a protective effect to the ALL cells by changes in MYC, LEF1, CCNDBP1, and GSK3b levels, which led to activation of the Wnt pathway and promoted leukemic cell proliferation in ALL[109]. In acute myeloid leukemia (AML), it has been suggested that AML-MSCs have dysregulation of the canonical Wnt signaling pathway, in which there is a decrease in $\beta$-catenin/TCF-LEF complex formation and decreased bone morphogenetic protein 4 (BMP4) expression[110]. MSC-derived WNT5a inhibits proliferation and promotes differentiation of HL60, an AML cell line, via activation of the non-canonical Wnt signaling pathway due to significantly increased expression of Ror2 and Calcium/calmodulin-dependent protein kinase II (CaMKII) and decreased expression of $\beta$-catenin and cyclin D1[111]. Healthy donor hematopoietic stem/progenitor cells (HSPCs) co-cultured on MSCs derived from Fanconi anemia patients with AML showed reduced secretion of prostaglandins (PGs) by mesenchymal inhibition of COX2 and led to decreased expression of NR4A transcription factors and $\beta$-catenin, representing a novel COX2/PG/ 
NR4A/Wnt signaling axis[112]. In summary, Wnt signaling is implicated in MSC function in development and cancer progression and can significantly remodel the TME to promote oncogenesis.

\section{Targeting Wnt Signaling}

New agents that target Wnt signaling are emerging in research. For example, there are porcupine (PORCN) inhibitors (e.g., LGK974 or WNT974, IWP-L6, CGX1321, ETC1922159, RXC004), AXIN1 activators (e.g., XAV939, niclosamide), Dickkopf1(DKK1) antibodies (e.g., DKN-01,CKAP4), FZD receptors inhibitors (e.g., OMP-18R5, OMP54F28), CBP/ $\beta$-catenin inhibitors (e.g., PRI-724,ICG-001) and a WNT5A mimic (Foxy-5)[2, 113]. Table 1 lists current Wnt modulators for cancer therapy in clinical trials from the U.S. National Library of Medicine database (https://www.clinicaltrials.gov/ access on June 23, 2019).

\subsection{PORCN Inhibitors}

PORCN inhibitors block secretion of Wnt by inhibiting palmitoylation in the TME. The efficiency of LGK974 was determined in different tumors such as squamous cell carcinoma[114],colorectal cancer[115], prostate cancer[116] and chronic myeloid leukemia (CML)[117]. However, LGK974 has low solubility and high toxicity in different tissues, a potent cyclodextrins: LGK974 complex was investigated in lung cancer[118]. WNT974in combination with a tyrosine kinase inhibitor(TKI) enhanced the targeting effect of CML stem and progenitor cells[117].IWP-L6 blocked the Wnt-LRP5/6 pathway and delayed tumor growth via the promotion of a strong tumor-specific $\mathrm{T}$ cell responseby regulating DC function[46].

\subsection{AXIN1 Activators}

AXIN1 activators play a role in the induction of $\beta$-catenin degradation. Niclosamide, an FDA-approved anthelmintic drug, eradicated cancer stemness and elicited therapeutic effects on colorectal cancer via disruption of the LEF1/DCLK1-B axis[119]. However, due to the low solubility, bioavailability and systemic exposure of niclosamide, niclosamide conjugated polypeptide nanoparticles were introduced for study in colon cancer[120]. Repression of the microRNA miR-31-5p suppressed proliferation, invasion and tumorigenesis of osteosarcoma cells via promoting AXIN1[121].

\subsection{DKK1 antibodies}

Although DKK1 is a secreted inhibitor of $\beta$-catenin-dependent Wnt signaling, DKK1 appears to increase tumor growth and metastasis in preclinical models due to activation of $\beta$-catenin-independent Wnt signaling and mediation of immunosuppressive TME and immune evasion[122].A monoclonal antibody $(\mathrm{mAb})$ against cytoskeleton-associated protein 4 (CKAP4), a novel DKK1 receptor mAbs suppressed xenograft tumor formation and extended the survival of pancreatic ductal adenocarcinoma mice [123]. Moreover, Dickkopfrelated protein 2 (DKK2), anantagonist of $\mathrm{Wnt} / \beta$-catenin signaling, suppressed tumor cell migration by reversing EndMT and downregulating stem cell markers in breast cancer[124]. 


\subsection{FZD Receptors Inhibitors}

Wnt receptor decoys function to prevent Wnt binding to FZD receptors. A phase $1 \mathrm{~b}$ dose escalation study of ipafricept (OMP54F28), a recombinant protein that inhibits Wnt signaling, in platinum-sensitive ovarian cancer was recently released, and the results indicated that bone toxicity at efficacy doses limited its use in ovarian cancer[125].

\subsection{CBP/ $\beta$-catenin Inhibitors}

$\mathrm{CBP} / \beta$-catenin inhibitors disrupt the interaction between $\mathrm{CBP}$ and $\beta$-catenin. One inhibitor, ICG-001, partially reversed EndMT and attenuated cancer stemness[126]. In addition, ICG-001 suppressed pancreatic cancer growth and significantly prolonged survival in an in vivo pancreatic ductal adenocarcinoma xenograft mouse model[127]. ICG-001 significantly prolonged the survival of NOD/SCID mice engrafted with drug-resistant primary ALL[128], and inhibited colony formation in sorted CD34+ CML progenitors in combination with imatinibmesylate[129].Moreover, inhibition by the $\mathrm{CBP} / \beta$-catenin antagonist C-82/PRI-724and the use of FLT3 TKI decreased Wnt/ $\beta$-catenin signaling in FLT3-mutant AML[130].

\subsection{WNT5A Mimic}

In addition, WNT5A upregulated the durable expression and activity of the indoleamine 2,3dioxygenase-1 (IDO) enzyme by local DCs and induced immunotolerance[131]. WNT5A is an $\beta$-catenin-independent ligand that has been shown to induce tumor suppression [132]. Foxy-5, a WNT5A-mimicking peptide, which has been recently used in clinical trials, significantly inhibited the initial metastatic dissemination of prostate cancer cells with absent or low WNT5A expression[133]. In human colonic cancer, the number of colonic CSCs was decreased by Foxy-5[134]. .

\section{Concluding Remarks}

Herein, we have systemically reviewed the complex relationship between Wnt signaling and many factors in the TME (Figure 2). As discussed, most research has focused on canonical Wnt signaling which is based on Wnt/ $\beta$-catenin interaction. Meanwhile, Wnt signaling plays an important role in sustaining the self-renewal potential of CSCs as well as LSCs, and subsequently enhances CSCs promotion. The immune cells in the TME mediate theimmune tolerance and immune evasion of cancer cells via Wnt signaling. In addition, Wnt signaling regulates ECM remodeling and causes overproduction of extracellular proteins in the TME to protect cancer cells from eradication. The transformation of stromal normal fibroblasts into the CAF phenotype has been implicated in promoting primary tumor growth and progression to metastatic disease. ECs in the TME induce tumor angiogenesis through Wnt signaling activation. There are several potential Wnt signaling modulator targets including but not limited to PORCN inhibitors, AXIN1 activators, Dickkopf1 antibodies, Wnt receptor decoys, CBP/ $\beta$-catenin inhibitors and a WNT5A mimic. However, Wnt signaling also plays a crucial homeostatic role in normal cells and restricts the administration of potent Wnt signaling inhibitors due to off-target toxicities. In the future, the dual targeting of Wnt signaling and the TME may efficiently eradicate cancer cells. Taken together, Wnt signaling 
tightly correlates to the TME, and targeting the Wnt signaling pathway in the TME is a promising tumor therapeutic strategy.

\section{References}

1. Nusse R and Clevers H, Wnt/beta-Catenin Signaling, Disease, and Emerging Therapeutic Modalities. Cell, 2017. 169(6): p. 985-999. [PubMed: 28575679]

2. Galluzzi L, et al., WNT Signaling in Cancer Immunosurveillance. Trends Cell Biol, 2019. 29(1): p. 44-65. [PubMed: 30220580]

3. Goldsberry WN, et al., A Review of the Role of Wnt in Cancer Immunomodulation. Cancers (Basel), 2019. 11(6).

4. Wu T and Dai Y, Tumor microenvironment and therapeutic response. Cancer Lett, 2017. 387: p. 61-68. [PubMed: 26845449]

5. Kahn M, Can we safely target the WNT pathway?Nat Rev Drug Discov, 2014. 13(7): p. 513-32. [PubMed: 24981364]

6. Duchartre Y, Kim YM, and Kahn M, The Wnt signaling pathway in cancer. Crit Rev Oncol Hematol, 2016. 99: p. 141-9. [PubMed: 26775730]

7. Alok A, et al., Wnt proteins synergize to activate beta-catenin signaling. J Cell Sci, 2017. 130(9): p. 1532-1544. [PubMed: 28289266]

8. Stamos JL and Weis WI, The beta-catenin destruction complex. Cold Spring Harb Perspect Biol, 2013. 5(1): p. a007898. [PubMed: 23169527]

9. Schulte G, Frizzleds and WNT/beta-catenin signaling--The black box of ligand-receptor selectivity, complex stoichiometry and activation kinetics. Eur J Pharmacol, 2015. 763(Pt B): p. 191-5. [PubMed: 26003275]

10. Janda CY, et al., Surrogate Wnt agonists that phenocopy canonical Wnt and beta-catenin signalling. Nature, 2017. 545(7653): p. 234-237. [PubMed: 28467818]

11. Daniels DL and Weis WI, Beta-catenin directly displaces Groucho/TLE repressors from Tcf/Lef in Wnt-mediated transcription activation. Nat Struct Mol Biol, 2005. 12(4): p. 364-71. [PubMed: 15768032]

12. Prasetyanti PR, et al., Regulation of stem cell self-renewal and differentiation by Wnt and Notch are conserved throughout the adenoma-carcinoma sequence in the colon. Mol Cancer, 2013. 12(1): p. 126. [PubMed: 24144042]

13. Kim JH, et al., Roles of Wnt Target Genes in the Journey of Cancer Stem Cells. Int J Mol Sci, 2017. 18(8).

14. De A, Wnt/Ca2+ signaling pathway: a brief overview. Acta Biochim Biophys Sin (Shanghai), 2011. 43(10): p. 745-56. [PubMed: 21903638]

15. Stolz A, et al., Wnt-mediated protein stabilization ensures proper mitotic microtubule assembly and chromosome segregation. EMBO Rep, 2015. 16(4): p. 490-9. [PubMed: 25656539]

16. Beck B and Blanpain C, Unravelling cancer stem cell potential. Nat Rev Cancer, 2013. 13(10): p. 727-38. [PubMed: 24060864]

17. Reya T and Clevers H, Wnt signalling in stem cells and cancer. Nature, 2005. 434(7035): p. 843-50. [PubMed: 15829953]

18. Chattopadhyay S, Chaklader M, and Law S, Aberrant Wnt Signaling Pathway in the Hematopoietic Stem/Progenitor Compartment in Experimental Leukemic Animal. J Cell Commun Signal, 2019. 13(1): p. 39-52. [PubMed: 29978347]

19. Staal FJ, et al., Aberrant Wnt Signaling in Leukemia. Cancers (Basel), 2016. 8(9).

20. Zhan T, Rindtorff N, and Boutros M, Wnt signaling in cancer. Oncogene, 2017. 36(11): p. 14611473. [PubMed: 27617575]

21. Park JI, et al., Telomerase modulates Wnt signalling by association with target gene chromatin. Nature, 2009. 460(7251): p. 66-72. [PubMed: 19571879]

22. Vijay GV, et al., GSK3beta regulates epithelial-mesenchymal transition and cancer stem cell properties in triple-negative breast cancer. Breast Cancer Res, 2019. 21(1): p. 37. [PubMed: 30845991] 
23. Yang J, et al., Angiomotin-p130 inhibits beta-catenin stability by competing with Axin for binding to tankyrase in breast cancer. Cell Death Dis, 2019. 10(3): p. 179. [PubMed: 30792381]

24. Domenici G, et al., A Sox2-Sox9 signalling axis maintains human breast luminal progenitor and breast cancer stem cells. Oncogene, 2019. 38(17): p. 3151-3169. [PubMed: 30622340]

25. Carmon KS, et al., LGR5 interacts and cointernalizes with Wnt receptors to modulate Wnt/betacatenin signaling. Mol Cell Biol, 2012. 32(11): p. 2054-64. [PubMed: 22473993]

26. Schepers AG, et al., Lineage tracing reveals Lgr5+ stem cell activity in mouse intestinal adenomas. Science, 2012. 337(6095): p. 730-5. [PubMed: 22855427]

27. Fu T, et al., FXR Regulates Intestinal Cancer Stem Cell Proliferation. Cell, 2019. 176(5): p. 10981112 e18. [PubMed: 30794774]

28. Wang LQ, et al., miR-372 and miR-373 enhance the stemness of colorectal cancer cells by repressing differentiation signaling pathways. Mol Oncol, 2018. 12(11): p. 1949-1964. [PubMed: 30171794]

29. Cao HZ, et al., LGR5 promotes cancer stem cell traits and chemoresistance in cervical cancer. Cell Death Dis, 2017. 8(9): p. e3039. [PubMed: 28880275]

30. Mei Y, et al., RIF1 promotes tumor growth and cancer stem cell-like traits in NSCLC by protein phosphatase 1-mediated activation of Wnt/beta-catenin signaling. Cell Death Dis, 2018. 9(10): p. 942. [PubMed: 30237512]

31. Gong L, et al., Serine-arginine protein kinase 1 promotes a cancer stem cell-like phenotype through activation of Wnt/beta-catenin signalling in NSCLC. J Pathol, 2016. 240(2): p. 184-96. [PubMed: 27391422]

32. Chua HH, et al., RBMY, a novel inhibitor of glycogen synthase kinase 3beta, increases tumor stemness and predicts poor prognosis of hepatocellular carcinoma. Hepatology, 2015. 62(5): p. 1480-96. [PubMed: 26185016]

33. Schreiber RD, Old LJ, and Smyth MJ, Cancer immunoediting: integrating immunity's roles in cancer suppression and promotion. Science, 2011. 331(6024): p. 1565-70. [PubMed: 21436444]

34. Thorsson V, et al., The Immune Landscape of Cancer. Immunity, 2018. 48(4): p. 812-830 e14. [PubMed: 29628290]

35. Staal FJ, Luis TC, and Tiemessen MM, WNT signalling in the immune system: WNT is spreading its wings. Nat Rev Immunol, 2008. 8(8): p. 581-93. [PubMed: 18617885]

36. Zhao DM, et al., Constitutive activation of Wnt signaling favors generation of memory CD8 T cells. J Immunol, 2010. 184(3): p. 1191-9. [PubMed: 20026746]

37. Forget MA, et al., Stimulation of Wnt/ss-catenin pathway in human CD8+ T lymphocytes from blood and lung tumors leads to a shared young/memory phenotype. PLoS One, 2012. 7(7): p. e41074. [PubMed: 22859966]

38. Driessens G, et al., Beta-catenin inhibits T cell activation by selective interference with linker for activation of T cells-phospholipase C-gamma1 phosphorylation. J Immunol, 2011. 186(2): p. 784-90. [PubMed: 21149602]

39. Ma J, et al., beta-catenin/TCF-1 pathway in T cell development and differentiation. $\mathbf{J}$ Neuroimmune Pharmacol, 2012. 7(4): p. 750-62. [PubMed: 22535304]

40. Jeevan-Raj B, et al., The Transcription Factor Tcf1 Contributes to Normal NK Cell Development and Function by Limiting the Expression of Granzymes. Cell Rep, 2017. 20(3): p. 613-626. [PubMed: 28723565]

41. Kling JC, et al., Temporal Regulation of Natural Killer T Cell Interferon Gamma Responses by beta-Catenin-Dependent and -Independent Wnt Signaling. Front Immunol, 2018. 9: p. 483. [PubMed: 29616022]

42. Zhou J, et al., Notch and wingless signaling cooperate in regulation of dendritic cell differentiation. Immunity, 2009. 30(6): p. 845-59. [PubMed: 19523851]

43. Fu C, et al., beta-Catenin in dendritic cells exerts opposite functions in cross-priming and maintenance of CD8+ T cells through regulation of IL-10. Proc Natl Acad Sci U S A, 2015. 112(9): p. 2823-8. [PubMed: 25730849]

44. Swafford D and Manicassamy S, Wnt signaling in dendritic cells: its role in regulation of immunity and tolerance. Discov Med, 2015. 19(105): p. 303-10. [PubMed: 25977193] 
45. Baur AS, et al., Denileukin diftitox (ONTAK) induces a tolerogenic phenotype in dendritic cells and stimulates survival of resting Treg. Blood, 2013. 122(13): p. 2185-94. [PubMed: 23958949]

46. Hong Y, et al., Deletion of LRP5 and LRP6 in dendritic cells enhances antitumor immunity. Oncoimmunology, 2016. 5(4): p. e1115941. [PubMed: 27141399]

47. Zhao F, et al., Paracrine Wnt5a-beta-Catenin Signaling Triggers a Metabolic Program that Drives Dendritic Cell Tolerization. Immunity, 2018. 48(1): p. 147-160 e7. [PubMed: 29343435]

48. Keerthivasan S, et al., beta-Catenin promotes colitis and colon cancer through imprinting of proinflammatory properties in T cells. Sci Transl Med, 2014. 6(225): p. $225 \mathrm{ra} 28$.

49. Ribas A and Wolchok JD, Cancer immunotherapy using checkpoint blockade. Science, 2018. 359(6382): p. 1350-1355. [PubMed: 29567705]

50. Spranger S, Bao R, and Gajewski TF, Melanoma-intrinsic beta-catenin signalling prevents antitumour immunity. Nature, 2015. 523(7559): p. 231-5. [PubMed: 25970248]

51. Taylor A, Rothstein D, and Rudd CE, Small-Molecule Inhibition of PD-1 Transcription Is an Effective Alternative to Antibody Blockade in Cancer Therapy. Cancer Res, 2018. 78(3): p. 706717. [PubMed: 29055015]

52. Harding JJ, et al., Prospective Genotyping of Hepatocellular Carcinoma: Clinical Implications of Next-Generation Sequencing for Matching Patients to Targeted and Immune Therapies. Clin Cancer Res, 2019. 25(7): p. 2116-2126. [PubMed: 30373752]

53. Luke JJ, et al., WNT/beta-catenin Pathway Activation Correlates with Immune Exclusion across Human Cancers. Clin Cancer Res, 2019. 25(10): p. 3074-3083. [PubMed: 30635339]

54. D'Amico L, et al., Dickkopf-related protein 1 (Dkk1) regulates the accumulation and function of myeloid derived suppressor cells in cancer. J Exp Med, 2016. 213(5): p. 827-40. [PubMed: 27045006]

55. Erdogan B and Webb DJ, Cancer-associated fibroblasts modulate growth factor signaling and extracellular matrix remodeling to regulate tumor metastasis. Biochem Soc Trans, 2017. 45(1): p. 229-236. [PubMed: 28202677]

56. Liu J, et al., Cancer-associated fibroblasts promote hepatocellular carcinoma metastasis through chemokine-activated hedgehog and TGF-beta pathways. Cancer Lett, 2016. 379(1): p. 49-59. [PubMed: 27216982]

57. Du J, et al., Extracellular matrix stiffness dictates Wnt expression through integrin pathway. Sci Rep, 2016. 6: p. 20395. [PubMed: 26854061]

58. Liu S, et al., Sirt1 regulates apoptosis and extracellular matrix degradation in resveratrol-treated osteoarthritis chondrocytes via the Wnt/beta-catenin signaling pathways. Exp Ther Med, 2017. 14(5): p. 5057-5062. [PubMed: 29201214]

59. Zhang R, et al., E6/E7-P53-POU2F1-CTHRC1 axis promotes cervical cancer metastasis and activates Wnt/PCP pathway. Sci Rep, 2017. 7: p. 44744. [PubMed: 28303973]

60. Huang $X$, et al., Wnt7a activates canonical Wnt signaling, promotes bladder cancer cell invasion, and is suppressed by miR-370-3p. J Biol Chem, 2018. 293(18): p. 6693-6706. [PubMed: 29549123]

61. Meng C, et al., MRTF-A mediates the activation of COL1A1 expression stimulated by multiple signaling pathways in human breast cancer cells. Biomed Pharmacother, 2018. 104: p. 718-728. [PubMed: 29807221]

62. He H, et al., The Wnt-beta-catenin signaling regulated MRTF-A transcription to activate migrationrelated genes in human breast cancer cells. Oncotarget, 2018. 9(20): p. 15239-15251. [PubMed: 29632640]

63. Wang Y, et al., Myosin Heavy Chain 10 (MYH10) Gene Silencing Reduces Cell Migration and Invasion in the Glioma Cell Lines U251, T98G, and SHG44 by Inhibiting the Wnt/beta-Catenin Pathway. Med Sci Monit, 2018. 24: p. 9110-9119. [PubMed: 30552850]

64. Li Y, et al., Oncolytic Ad co-expressing decorin and Wnt decoy receptor overcomes chemoresistance of desmoplastic tumor through degradation of ECM and inhibition of EMT. Cancer Lett, 2019. 459: p. 15-29. [PubMed: 31150821]

65. Na Y, et al., Potent antitumor effect of neurotensin receptor-targeted oncolytic adenovirus co-expressing decorin and Wnt antagonist in an orthotopic pancreatic tumor model. J Control Release, 2015. 220(Pt B): p. 766-82. [PubMed: 26471393] 
66. Hawkins AG, et al., The Ewing Sarcoma Secretome and Its Response to Activation of Wnt/betacatenin Signaling. Mol Cell Proteomics, 2018. 17(5): p. 901-912. [PubMed: 29386236]

67. McCarthy JB, El-Ashry D, and Turley EA, Hyaluronan, Cancer-Associated Fibroblasts and the Tumor Microenvironment in Malignant Progression. Front Cell Dev Biol, 2018. 6: p. 48. [PubMed: 29868579]

68. Sasaki S, et al., Crucial involvement of the CCL3-CCR5 axis-mediated fibroblast accumulation in colitis-associated carcinogenesis in mice. Int J Cancer, 2014. 135(6): p. 1297-306. [PubMed: 24510316]

69. Lorsy E, et al., Loss of Dickkopf 3 Promotes the Tumorigenesis of Basal Breast Cancer. PLoS One, 2016. 11 (7): p. e0160077. [PubMed: 27467270]

70. Caffo M, et al., Modulation of Dkk-3 and claudin-5 as new therapeutic strategy in the treatment of meningiomas. Oncotarget, 2017. 8(40): p. 68280-68290. [PubMed: 28978116]

71. Ferreira Tojais N, et al., Frizzled7 controls vascular permeability through the Wnt-canonical pathway and cross-talk with endothelial cell junction complexes. Cardiovasc Res, 2014. 103(2): p. 291-303. [PubMed: 24866384]

72. Ferrari N, et al., Dickkopf-3 links HSF1 and YAP/TAZ signalling to control aggressive behaviours in cancer-associated fibroblasts. Nat Commun, 2019. 10(1): p. 130. [PubMed: 30631061]

73. Hu JL, et al., CAFs secreted exosomes promote metastasis and chemotherapy resistance by enhancing cell stemness and epithelial-mesenchymal transition in colorectal cancer. Mol Cancer, 2019. 18(1): p. 91. [PubMed: 31064356]

74. Izumi D, et al., TIAM1 promotes chemoresistance and tumor invasiveness in colorectal cancer. Cell Death Dis, 2019. 10(4): p. 267. [PubMed: 30890693]

75. Niell N, et al., The human PKP2/plakophilin-2 gene is induced by Wnt/beta-catenin in normal and colon cancer-associated fibroblasts. Int J Cancer, 2018. 142(4): p. 792-804. [PubMed: 29044515]

76. Kramer N, et al., Autocrine WNT2 signaling in fibroblasts promotes colorectal cancer progression. Oncogene, 2017. 36(39): p. 5460-5472. [PubMed: 28553956]

77. Yu B, et al., Periostin secreted by cancer-associated fibroblasts promotes cancer stemness in head and neck cancer by activating protein tyrosine kinase 7. Cell Death Dis, 2018. 9(11): p. 1082. [PubMed: 30348980]

78. Rohwer N, et al., Non-canonical HIF-1 stabilization contributes to intestinal tumorigenesis. Oncogene, 2019.

79. Rupp C, et al., IGFBP7, a novel tumor stroma marker, with growth-promoting effects in colon cancer through a paracrine tumor-stroma interaction. Oncogene, 2015. 34(7): p. 815-25. [PubMed: 24632618]

80. Munoz-Galvan S, et al., Tumor cell-secreted PLD increases tumor stemness by senescencemediated communication with microenvironment. Oncogene, 2019. 38(8): p. 1309-1323. [PubMed: 30305726]

81. Horman SR, et al., Functional profiling of microtumors to identify cancer associated fibroblastderived drug targets. Oncotarget, 2017. 8(59): p. 99913-99930. [PubMed: 29245949]

82. Valenti G, et al., Cancer Stem Cells Regulate Cancer-Associated Fibroblasts via Activation of Hedgehog Signaling in Mammary Gland Tumors. Cancer Res, 2017. 77(8): p. 2134-2147. [PubMed: 28202523]

83. Zhao L, et al., An integrated analysis identifies STAT4 as a key regulator of ovarian cancer metastasis. Oncogene, 2017. 36(24): p. 3384-3396. [PubMed: 28114283]

84. Avgustinova A, et al., Tumour cell-derived Wnt7a recruits and activates fibroblasts to promote tumour aggressiveness. Nat Commun, 2016. 7: p. 10305. [PubMed: 26777421]

85. Kaur A, et al., sFRP2 in the aged microenvironment drives melanoma metastasis and therapy resistance. Nature, 2016. 532(7598): p. 250-4. [PubMed: 27042933]

86. Chen Y, et al., Aberrant low expression of p85alpha in stromal fibroblasts promotes breast cancer cell metastasis through exosome-mediated paracrine Wnt10b. Oncogene, 2017. 36(33): p. 46924705. [PubMed: 28394344]

87. Tang S, et al., Oxidized ATM promotes abnormal proliferation of breast CAFs through maintaining intracellular redox homeostasis and activating the PI3K-AKT, MEK-ERK, and Wnt-beta-catenin signaling pathways. Cell Cycle, 2015. 14(12): p. 1908-24. [PubMed: 25970706] 
88. Piera-Velazquez S and Jimenez SA, Endothelial to Mesenchymal Transition: Role in Physiology and in the Pathogenesis of Human Diseases. Physiol Rev, 2019. 99(2): p. 1281-1324. [PubMed: 30864875]

89. Pinto MT, et al., Endothelial cells from different anatomical origin have distinct responses during SNAIL/TGF-beta2-mediated endothelial-mesenchymal transition. Am J Transl Res, 2018. 10(12): p. 4065-4081. [PubMed: 30662651]

90. Wang SH, et al., Tumour cell-derived WNT5B modulates in vitro lymphangiogenesis via induction of partial endothelial-mesenchymal transition of lymphatic endothelial cells. Oncogene, 2017. 36(11): p. 1503-1515. [PubMed: 27593938]

91. Scholz B, et al., Endothelial RSPO3 Controls Vascular Stability and Pruning through Noncanonical WNT/Ca(2+)/NFAT Signaling. Dev Cell, 2016. 36(1): p. 79-93. [PubMed: 26766444]

92. Rapp J, et al., Increased Wnt5a in squamous cell lung carcinoma inhibits endothelial cell motility. BMC Cancer, 2016. 16(1): p. 915. [PubMed: 27876017]

93. Shi YN, et al., Wnt5a and its signaling pathway in angiogenesis. Clin Chim Acta, 2017. 471: p. 263-269. [PubMed: 28641961]

94. Griveau A, et al., A Glial Signature and Wnt7 Signaling Regulate Glioma-Vascular Interactions and Tumor Microenvironment. Cancer Cell, 2018. 33(5): p. 874-889 e7. [PubMed: 29681511]

95. Al Thawadi H, et al., VE-cadherin cleavage by ovarian cancer microparticles induces beta-catenin phosphorylation in endothelial cells. Oncotarget, 2016. 7(5): p. 5289-305. [PubMed: 26700621]

96. Guo P, et al., Ribosomal protein S15a promotes tumor angiogenesis via enhancing Wnt/betacatenin-induced FGF18 expression in hepatocellular carcinoma. Oncogene, 2018. 37(9): p. 12201236. [PubMed: 29242604]

97. Peterson YK, et al., Frizzled-5: a high affinity receptor for secreted frizzled-related protein-2 activation of nuclear factor of activated T-cells $\mathrm{c} 3$ signaling to promote angiogenesis. Angiogenesis, 2017. 20(4): p. 615-628. [PubMed: 28840375]

98. Huang Z and Feng Y, Exosomes Derived From Hypoxic Colorectal Cancer Cells Promote Angiogenesis Through Wnt4-Induced beta-Catenin Signaling in Endothelial Cells. Oncol Res, 2017. 25(5): p. 651-661. [PubMed: 27712599]

99. Vallee A, Guillevin R, and Vallee JN, Vasculogenesis and angiogenesis initiation under normoxic conditions through Wnt/beta-catenin pathway in gliomas. Rev Neurosci, 2018. 29(1): p. 71-91. [PubMed: 28822229]

100. Linke F, et al., Microenvironmental interactions between endothelial and lymphoma cells: a role for the canonical WNT pathway in Hodgkin lymphoma. Leukemia, 2017. 31(2): p. 361-372. [PubMed: 27535218]

101. Yan TL, et al., Up-regulation of syncytin-1 contributes to TNF-alpha-enhanced fusion between OSCC and HUVECs partly via Wnt/beta-catenin-dependent pathway. Sci Rep, 2017. 7: p. 40983. [PubMed: 28112190]

102. Bassett EA, et al., Norrin/Frizzled4 signalling in the preneoplastic niche blocks medulloblastoma initiation. Elife, 2016. 5.

103. Planutis K, Planutiene M, and Holcombe RF, A novel signaling pathway regulates colon cancer angiogenesis through Norrin. Sci Rep, 2014. 4: p. 5630. [PubMed: 25005225]

104. Yin T and Li L, The stem cell niches in bone. J Clin Invest, 2006. 116(5): p. 1195-201. [PubMed: 16670760]

105. Huang X, et al., The Effects of the WNT-Signaling Modulators BIO and PKF118-310 on the Chondrogenic Differentiation of Human Mesenchymal Stem Cells. Int J Mol Sci, 2018. 19(2).

106. Eslaminejad MB, Karimi N, and Shahhoseini M, Chondrogenic differentiation of human bone marrow-derived mesenchymal stem cells treated by GSK-3 inhibitors. Histochem Cell Biol, 2013. 140(6): p. 623-33. [PubMed: 23839780]

107. Wu D and Pan W, GSK3: a multifaceted kinase in Wnt signaling. Trends Biochem Sci, 2010. 35(3): p. 161-8. [PubMed: 19884009]

108. Takiguchi G, et al., Wnt5a-Ror2 signaling in mesenchymal stem cells promotes proliferation of gastric cancer cells by activating CXCL16-CXCR6 axis. Cancer Sci, 2016. 107(3): p. 290-7. [PubMed: 26708384] 
109. Yang Y, et al., Wnt pathway contributes to the protection by bone marrow stromal cells of acute lymphoblastic leukemia cells and is a potential therapeutic target. Cancer Lett, 2013. 333(1): p. 9-17. [PubMed: 23333798]

110. Azevedo PL, et al., Canonical WNT Signaling Pathway is Altered in Mesenchymal Stromal Cells From Acute Myeloid Leukemia Patients And Is Implicated in BMP4 Down-Regulation. Transl Oncol, 2019. 12(4): p. 614-625. [PubMed: 30703678]

111. Shen YL, et al., Bone marrow mesenchymal stem cell-derived Wnt5a inhibits leukemia cell progression in vitro via activation of the non-canonical Wnt signaling pathway. Oncol Lett, 2014. 8(1): p. 85-90. [PubMed: 24959224]

112. Wu L, et al., Mesenchymal COX2-PG secretome engages NR4A-WNT signalling axis in haematopoietic progenitors to suppress anti-leukaemia immunity. Br J Haematol, 2018. 183(3): p. 445-456. [PubMed: 30106181]

113. Kim YM, Gang EJ, and Kahn M, CBP/Catenin antagonists: Targeting LSCs'Achilles heel. Exp Hematol, 2017. 52: p. 1-11. [PubMed: 28479420]

114. Zimmerli D, et al., WNT ligands control initiation and progression of human papillomavirusdriven squamous cell carcinoma. Oncogene, 2018. 37(27): p. 3753-3762. [PubMed: 29662191]

115. Picco G, et al., Loss of AXIN1 drives acquired resistance to WNT pathway blockade in colorectal cancer cells carrying RSPO3 fusions. EMBO Mol Med, 2017. 9(3): p. 293-303. [PubMed: 28100566]

116. Ma F, et al., SOX9 drives WNT pathway activation in prostate cancer. J Clin Invest, 2016. 126(5): p. 1745-58. [PubMed: 27043282]

117. Agarwal P, et al., Enhanced targeting of CML stem and progenitor cells by inhibition of porcupine acyltransferase in combination with TKI. Blood, 2017. 129(8): p. 1008-1020. [PubMed: 28011678]

118. Guimaraes PPG, et al., Potent in vivo lung cancer Wnt signaling inhibition via cyclodextrinLGK974 inclusion complexes. J Control Release, 2018. 290: p. 75-87. [PubMed: 30290244]

119. Park SY, et al., Inhibition of LEF1-Mediated DCLK1 by Niclosamide Attenuates Colorectal Cancer Stemness. Clin Cancer Res, 2019. 25(4): p. 1415-1429. [PubMed: 30446587]

120. Bhattacharyya J, et al., Niclosamide-conjugated polypeptide nanoparticles inhibit Wnt signaling and colon cancer growth. Nanoscale, 2017. 9(34): p. 12709-12717. [PubMed: 28828438]

121. Chen X, et al., Down-regulation of microRNA-31-5p inhibits proliferation and invasion of osteosarcoma cells through Wnt/beta-catenin signaling pathway by enhancing AXIN1. Exp Mol Pathol, 2019. 108: p. 32-41. [PubMed: 30844369]

122. Kagey $\mathrm{MH}$ and $\mathrm{He} \mathrm{X}$, Rationale for targeting the Wnt signalling modulator Dickkopf-1 for oncology. Br J Pharmacol, 2017. 174(24): p. 4637-4650. [PubMed: 28574171]

123. Kimura H, et al., CKAP4, a DKK1 Receptor, Is a Biomarker in Exosomes Derived from Pancreatic Cancer and a Molecular Target for Therapy. Clin Cancer Res, 2019. 25(6): p. 19361947. [PubMed: 30610103]

124. Mu J, et al., Dickkopf-related protein 2 induces G0/G1 arrest and apoptosis through suppressing Wnt/beta-catenin signaling and is frequently methylated in breast cancer. Oncotarget, 2017. 8(24): p. 39443-39459. [PubMed: 28467796]

125. Moore KN, et al., A phase 1b dose escalation study of ipafricept (OMP54F28) in combination with paclitaxel and carboplatin in patients with recurrent platinum-sensitive ovarian cancer. Gynecol Oncol, 2019.

126. Chen Y, et al., TNF-alpha derived from M2 tumor-associated macrophages promotes epithelial-mesenchymal transition and cancer stemness through the Wnt/beta-catenin pathway in SMMC-7721 hepatocellular carcinoma cells. Exp Cell Res, 2019. 378(1): p. 41-50. [PubMed: 30844387]

127. Arensman MD, et al., The CREB-binding protein inhibitor ICG-001 suppresses pancreatic cancer growth. Mol Cancer Ther, 2014. 13(10): p. 2303-14. [PubMed: 25082960]

128. Gang EJ, et al., Small-molecule inhibition of CBP/catenin interactions eliminates drug-resistant clones in acute lymphoblastic leukemia. Oncogene, 2014. 33(17): p. 2169-78. [PubMed: 23728349] 
129. Kim YM, et al., The gamma catenin/CBP complex maintains survivin transcription in betacatenin deficient/depleted cancer cells. Curr Cancer Drug Targets, 2011.11 (2): p. 213-25. [PubMed: 21158719]

130. Jiang X, et al., Disruption of Wnt/beta-Catenin Exerts Antileukemia Activity and Synergizes with FLT3 Inhibition in FLT3-Mutant Acute Myeloid Leukemia. Clin Cancer Res, 2018. 24(10): p. 2417-2429. [PubMed: 29463558]

131. Holtzhausen A, et al., Melanoma-Derived Wnt5a Promotes Local Dendritic-Cell Expression of IDO and Immunotolerance: Opportunities for Pharmacologic Enhancement of Immunotherapy. Cancer Immunol Res, 2015. 3(9): p. 1082-95. [PubMed: 26041736]

132. Prasad CP, et al., WNT5A as a therapeutic target in breast cancer. Cancer Metastasis Rev, 2018. 37(4): p. 767-778. [PubMed: 30171384]

133. Canesin G, et al., Treatment with the WNT5A-mimicking peptide Foxy-5 effectively reduces the metastatic spread of WNT5A-low prostate cancer cells in an orthotopic mouse model. PLoS One, 2017. 12(9): p. e0184418. [PubMed: 28886116]

134. Osman J, et al., The WNT5A Agonist Foxy5 Reduces the Number of Colonic Cancer Stem Cells in a Xenograft Mouse Model of Human Colonic Cancer. Anticancer Res, 2019. 39(4): p. 1719-1728. [PubMed: 30952711] 


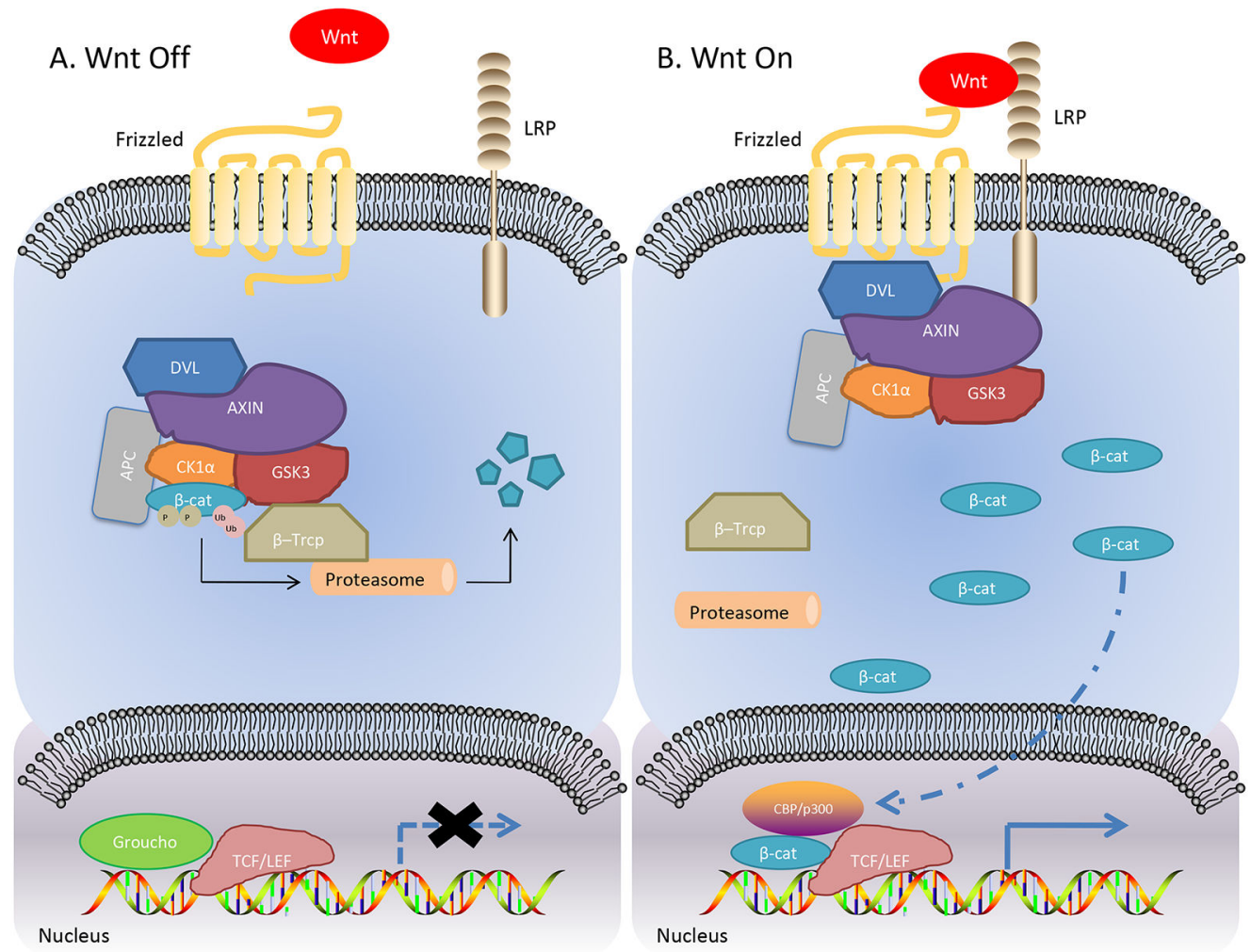

Figure 1. The Canonical Wnt Pathway.

A. Inactivation of Wnt signaling. B. Activation of Wnt signaling. LRP, lipoprotein receptorrelated protein; DVL, disheveled segment polarity protein; CK1a, casein kinase 1a; GSK-13, glycogen synthase kinase 3 ; $\beta$-cat, $\beta$-catenin; APC, adenomatous polyposis coli; TCF/LEF, T-cell Factor/Lymphoid Enhancer Factor; CBP, CREB-binding protein. 


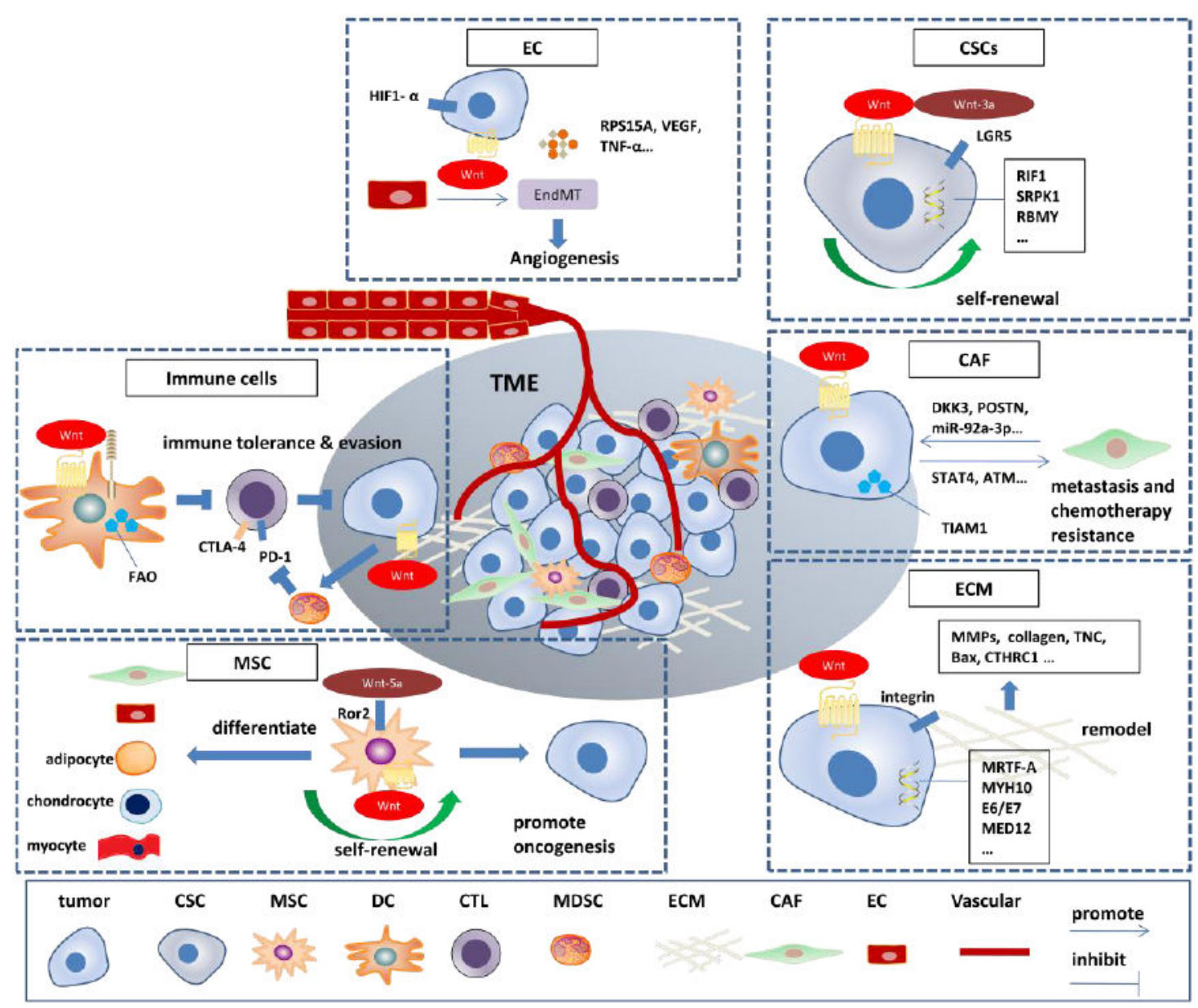

Figure 2. Wnt signaling in the tumor microenvironment (TME).

ECs, endothelial cells; EndMT, endothelial-mesenchymal transition; CSCs, cancer stem cells; LGR5, Leucine-rich repeat-containing G-protein-coupled receptor 5; ECM, extracellular matrix; CAF, Cancer associated fibroblast; TIAM1, T-lymphoma invasion and metastasis-inducing protein-1; FAO, fatty acid oxidation; CTLA-4, cytotoxic T lymphocyteassociated protein 4; PD-1, programmed cell death-1; CTL, cytotoxic T lymphocyte; MSC, mesenchymal stem cell; DC, dendritic cell; MDSC, myeloid-derived suppressor cell. 
Table1.

Clinical Trials of Wnt Modulators in cancer.

\begin{tabular}{|c|c|c|c|c|}
\hline Mechanism & Agent & Disease & Status & Identifier \\
\hline \multirow[t]{16}{*}{ PORCN inhibitor } & \multirow[t]{11}{*}{ WNT974/LGK974 } & Squamous Cell Carcinoma, Head And Neck & Withdrawn & NCT02649530 \\
\hline & & Metastatic Colorectal Cancer & Completed & NCT02278133 \\
\hline & & Pancreatic CancerBRAF Mutant & Recruiting & NCT01351103 \\
\hline & & Colorectal & & \\
\hline & & CancerMelanomaTriple Negative & & \\
\hline & & Breast CancerHead and Neck & & \\
\hline & & Squamous Cell CancerCervical & & \\
\hline & & Squamous Cell & & \\
\hline & & CancerEsophageal Squamous & & \\
\hline & & Cell CancerLung Squamous Cell & & \\
\hline & & Cancer & & \\
\hline & CGX1321 & Solid Tumors & Recruiting & NCT02675946 \\
\hline & & GI Cancer & & \\
\hline & $\mathrm{RXC004}$ & Cancer & Not yet recruiting & NCT03447470 \\
\hline & & Solid Tumor & & \\
\hline & ETC-1922159 & Solid Tumors & $\begin{array}{l}\text { Active, not } \\
\text { recruiting }\end{array}$ & NCT02521844 \\
\hline \multirow[t]{4}{*}{ Wnt-5a protein } & \multirow[t]{4}{*}{ Foxy-5 } & Colon Cancer & Recruiting & NCT03883802 \\
\hline & & Metastatic Breast Cancer & Completed & NCT02020291 \\
\hline & & Colorectal Cancer & & \\
\hline & & Prostate Cancer & & \\
\hline \multirow[t]{9}{*}{ DKK1 antibody } & \multirow[t]{9}{*}{ DKN-01 } & Hepatocellular Carcinoma & Recruiting & NCT03645980 \\
\hline & & Endometrial Cancer & Recruiting & NCT03395080 \\
\hline & & Uterine Cancer & & \\
\hline & & Ovarian Cancer & & \\
\hline & & Esophageal Neoplasms & Recruiting & NCT02013154 \\
\hline & & Adenocarcinoma of the Gastroesophageal Junction & & \\
\hline & & Gastroesophageal Cancer & & \\
\hline & & Squamous Cell Carcinoma & & \\
\hline & & Gastric Adenocarcinoma & & \\
\hline \multirow{2}{*}{$\begin{array}{l}\text { Wnt signaling pathway } \\
\text { inhibitor }\end{array}$} & SM08502 & Solid Tumor & Recruiting & NCT03355066 \\
\hline & XNW7201 & Advanced Solid Tumors & Not yet recruiting & NCT03901950 \\
\hline \multirow{11}{*}{$\begin{array}{l}\text { CBP/beta-catenin } \\
\text { Antagonist }\end{array}$} & \multirow[t]{5}{*}{ PRI-724 } & Colorectal Adenocarcinoma & Withdrawn & NCT02413853 \\
\hline & & Stage IVA Colorectal Cancer & & \\
\hline & & Stage IVB Colorectal Cancer & & \\
\hline & & Acute Myeloid Leukemia & Completed & NCT01606579 \\
\hline & & Chronic Myeloid Leukemia & & \\
\hline & CWP232291 & Acute Myeloid Leukemia & Completed & NCT01398462 \\
\hline & & Chronic Myelomonocytic & & \\
\hline & & Leukemia & & \\
\hline & & Myelodysplastic Syndrome & & \\
\hline & & Myelofibrosis & & \\
\hline & & Colon Cancer & & \\
\hline AXIN1 activator & Niclosamide & Colon Cancer & Recruiting & NCT02687009 \\
\hline Wnt signaling modulator & Resveratrol & Colon Cancer & Completed & NCT00256334 \\
\hline & & Cancer & & \\
\hline
\end{tabular}

